\title{
Three-year functional outcome after open pelvic fracture treatment: a retrospective case series from a level I trauma center
}

\author{
Yi-Hsun Yu ${ }^{1}$ (1) $\cdot$ Yung-Heng Hsu ${ }^{1} \cdot$ Ying-Chao Chou ${ }^{1} \cdot$ Chang-Heng Liu ${ }^{1} \cdot$ I.-Chuan Tseng ${ }^{1} \cdot$ I.-Jung Chen ${ }^{1}$
}

Received: 10 October 2021 / Accepted: 7 February 2022 / Published online: 28 February 2022

(c) The Author(s) 2022

\begin{abstract}
Purpose Open pelvic fractures have high mortality rates, and survivors may have ongoing functional deficits from severe trauma and invasive life-saving procedures. However, there are limited reports regarding the functional status evaluation following open pelvic fractures. We aimed to report the treatment experiences and short-term functional outcomes of patients with open pelvic fractures.

Methods We retrospectively reviewed the data of 19 consecutive patients with pelvic fractures who underwent treatment at a single institute between January 2014 and June 2018. The resuscitation protocol, osteosynthesis strategy, reduction quality of the pelvic ring, and functional outcomes were analyzed.

Results The incidence and mortality rates in patients with open pelvic fractures were 4.9 and $21.6 \%$, respectively. Ten, one, and seven of the open wounds related to the pelvic fractures were located in Faringer zones I, II, and III, respectively. Fractures of four patients were categorized as classes 1 and 2, and those of 11 patients as class 3, according to the Jones-Powell classification. Eleven of $19(57.9 \%)$ and 9 of 19 (47.5\%) revealed excellent reduction quality by Matta/Torenetta and Lefaivre criteria, respectively. The Merle d'Aubigné score improved at each evaluation but stagnated after 24 months. The Majeed hip score also improved at the 12-month evaluation but the improvement stopped thereafter. At a 3-year follow-up, the patients with excellent reduction of the pelvic ring showed the highest functional performances.

Conclusion Improvements in functional status of patients with open pelvic fractures can be anticipated based on the reduction quality of the pelvis ring.
\end{abstract}

Keywords Open pelvic fracture $\cdot$ Functional outcome $\cdot$ Retrospective $\cdot$ Treatment protocol

\section{Introduction}

Many pelvic fractures occur during high-energy activities. The pelvic fracture itself, or the concomitant injuries, may lead to life-threatening conditions and further long-term or permanent functional deficits [1-3]. Therefore, the timely and optimal management of such injuries is a challenge for orthopedic surgeons [4, 5].

With advancements in resuscitation procedures and improvements in the understanding of surgical anatomy as well as in surgical skills, a functional status following closed

Yi-Hsun Yu

alanyu1007@gmail.com

1 Department of Orthopedic Surgery, Musculoskeletal Research Center, Chang Gung Memorial Hospital and Chang Gung University, 5 Fu-Hsin Street, Kweishan, Tao-Yuan 33302, Taiwan pelvic fractures comparable to the pre-injury status may be achieved [6, 7]. However, major organ injuries in patients with open pelvic fractures may be systemic, thus making the functional recovery unpredictable. There are limited reports regarding the functional status evaluation following open pelvic fractures in the literature [4].

The current study aimed to report the experiences of patients with open pelvic fractures and the treatment protocols. The functional outcomes over an average follow-up duration of 36 months were also examined. Additionally, the etiologies that might be associated with worse functional outcomes were also studied. 


\section{Materials and methods}

The data and images of patients with open pelvic fractures visiting our institute between January 2014 and June 2018 were retrospectively collected from the registration database of the institute. Patients with (1) open pelvic fracture (2) osteosynthesis for the pelvic ring injury (3) age $\geq 18$ years, and (4) complete radiological and functional follow-ups were included. The patients who did not meet the inclusion criteria were excluded.

The data on age, gender, location of resuscitation, mechanism of injury, associated classifications for the open pelvic fracture, injury severity score (ISS), new injury severity score (NISS), numbers of surgery, follow-up duration, and time to union of the enrolled patients were reviewed and recorded.

\section{Resuscitation protocol}

The resuscitative protocols for patients were classified into two categories based on the type of pelvic fracture (closed or open, Fig. 1). For closed pelvic fractures, patients were resuscitated and managed according to our established protocol, which is primarily based on the Advanced Trauma Life Support Guidelines. If these patients developed shock, a blood transfusion with packed red blood cells, fresh frozen plasma, and platelets in a 1:1:1 ratio was provided. Furthermore, when they were unresponsive to fluids and blood resuscitation, arterial embolization (AE) was performed as the priority resuscitation procedure to stop retroperitoneal bleeding, in case of major bleeding originating from the pelvic fracture.

In contrast, in patients with open pelvic fractures, procedures such as surgical debridement, gauze packing of the open surgical wound, and external fixation in cases of pelvic instability were executed following primary resuscitation. The blood transfusion protocol was similar to that for closed pelvic fractures. Life-saving surgeries, such as thoracotomy and laparotomy, were performed simultaneously or sequentially during damage control orthopedic procedures in the operation theater. If the hemodynamic status remained unstable, AE was performed after life-saving procedures to facilitate hemostasis from the pelvic region. The packed gauzes were usually removed at $24 \mathrm{~h}$ after the surgery, and repeated debridement procedures were usually performed to decrease contamination. The timing for other surgeries was chosen based on the patient's condition. Pelvic osteosynthesis was performed as soon as possible after the patient had been hemodynamically stabilized.

While diversional colostomy was not a routine procedure for these patients, it was usually performed within $48 \mathrm{~h}$ after the trauma. The decision for diversional colostomy was collectively made by the traumatologist and the orthopedic traumatologist. Diversional colostomy was recommended in two circumstances: (1) for an open wound located at the perineum or the existence of rectal injury referring to Faringer zone I injury [8] and (2) the existence of bowel injury, for which the patient underwent bowel resection/anastomosis surgery, and the procedure of diversional colostomy was performed by the traumatologist.

\section{Fracture and associated classifications}

In addition to patients' demographic data, several classifications and scoring systems were used in this study. Trauma scores, such as the Injury Severity Score (ISS) and New Injury Severity Score (NISS), which are generally applied to patients after major blunt trauma were used [9, 10].

The Arbeitsgemeinschaft für Osteosynthesefragen (AO) classification for pelvic fracture was applied to determine the stability of pelvic ring injury [11]. This classification (a)

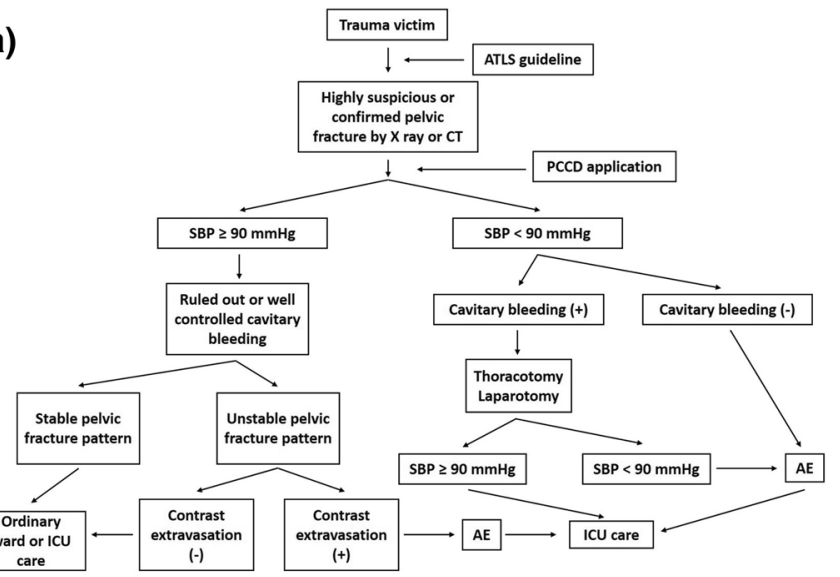

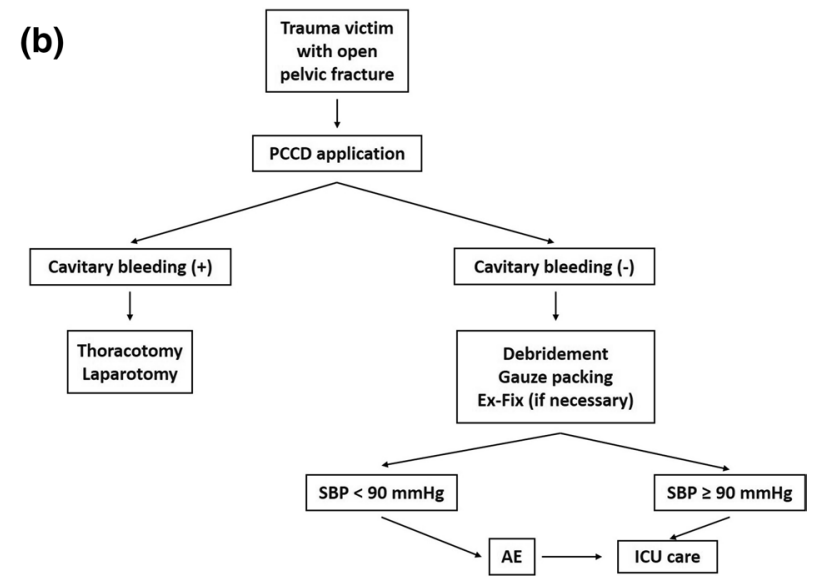

Fig. 1 Resuscitation protocol of patients with pelvic fracture. a Closed pelvic fracture. b Open pelvic fracture 
divides patients into three groups according to the severity and complexity of their injury: type A, stable pelvic ring injury; type B, partially unstable pelvic ring, and type C, completely unstable pelvic ring.

To represent the impact of open wounds related to pelvic fractures, Faringer and Jones-Powell classifications $[8,12]$, which focus solely on the location of open wounds and location of open wounds with fracture stability, respectively, were used specifically for open pelvic fractures. The Faringer classification was developed to classify open pelvic fracture by anatomical location of the wound: zone I (perineum, anterior pubis, medial buttock, and posterior sacrum), zone II (medial thigh and groin crease, and zone III (posterolateral buttock, iliac crest). The Jones-Powell is composed of the open wound site and fracture stability and classified into class 1 (stable pelvic ring), 2 (unstable pelvic ring without rectal or perineal wound), and 3 (unstable pelvic ring with rectal and/ or perineal wound).

\section{Radiological evaluation}

Radiological outcomes were determined through postoperative radiography using three standard pelvic radiographic views: anteroposterior, inlet, and outlet views. We applied the picture archiving and communication system to adjust the magnification of the area of interest. All radiographs were assessed using a similar method. Regarding the quality of post-osteosynthesis, we adapted the Matta/Tornetta and the Lefaivre criteria [13-15] to evaluate vertical displacement and pelvic symmetry, respectively (Table 1).

All images were primarily reviewed by two surgeons. If the surgeons had similar interpretations, the mean score was recorded. In the cases of different interpretations but similar scores, the differences were overlooked, and the scores were reported. However, if both surgeons provided different interpretations and scores, another senior surgeon (Y.-H, Y.) interpreted the images to determine the final score.

Table 1 Grading of radiological outcomes based on Matta/Tornetta and Lefaivre criteria [13]

\begin{tabular}{lll}
\hline & $\begin{array}{l}\text { Matta/Tornetta criteria dis- } \\
\text { placement }(\mathrm{mm})\end{array}$ & $\begin{array}{l}\text { Lefaivre criteria } \\
\text { displacement } \\
(\mathrm{mm})\end{array}$ \\
\hline Excellent & $<4$ & $<5$ \\
Good & $4-10$ & $5-10$ \\
Fair & $10-20$ & $10-20$ \\
Poor & $>20$ & $>20$ \\
\hline
\end{tabular}

\section{Rehabilitation protocol and functional outcome evaluations}

The rehabilitation protocol for these patients was individualized according to their concomitant injuries. Generally, patients were allowed to perform bedside rehabilitation exercises, such as rolling and passive/active muscular stretch exercises, once their pelvic ring injury had stabilized. When all injuries had stabilized, training for crutch- or wheelchairassisted ambulation was performed. There was no routine pharmaceutical prophylaxis for venous thromboembolism (VTE). However, VTE was mechanically prevented with the use of compression socks in each patient if there was no contraindication for such therapy (for example, cases such as degloving injury of the lower extremities). Functional outcome evaluations (Merle d'Aubigné score and Majeed hip score) were performed at 3,6 , and 12 months and thereafter annually after discharge from the hospital [16-19].

\section{Statistical analysis}

Statistical analyses were conducted using SPSS version 18.0 (SPSS Inc., Chicago, IL, USA). Continuous variables were reported as means \pm standard deviations and medians (ranges). Categorical variables were expressed as numbers (percentages). The cohort in this study was not normally distributed as found by Shapiro-Wilk test. Therefore, the study employed a nonparametric Mann-Whitney $U$ test for the analysis of continuous variables. A two-tailed $P$-value of 0.05 was considered statistically significant.

\section{Results}

During the study period, a total of 772 patients with pelvic fractures were primarily resuscitated or transferred to the emergency department (ED). Among these patients, 37 (4.9\%) were diagnosed with an open pelvic fracture. Eight patients failed to respond to resuscitation and died at the ED due to multiple injuries. Overall, the mortality rate for patients with pelvic fractures was $21.6 \%$.

After resuscitative procedures, 29 patients survived and were discharged after complete treatment courses. Of these, eight patients who did not undergo osteosynthesis (six patients refused surgical intervention because of the patients' or the relatives' consent, and two were too sick to be operated within the recommended time) and two who were lost to follow-up, were excluded. Finally, 19 patients were included in this study. All the 19 patients were operated on and cared by two surgeons (I-C. T. and Y.-H. Y.).

Demographic data of the patients are presented in Table 2. There were 10 male and 9 female individuals with a mean age of $37.5 \pm 17.8$ years. There were four, six, and 
Table 2 Demographic data of survivors with open pelvic fractures who completed treatment and follow-up courses

\begin{tabular}{|c|c|c|c|}
\hline & $N(\%)$ & Mean (SD) & Median (range) \\
\hline \multicolumn{4}{|l|}{ Sex } \\
\hline Male & $10(52.6)$ & NA & NA \\
\hline Female & $9(47.4)$ & NA & NA \\
\hline Age (years) & - & $37.5(17.8)$ & $35(6-74)$ \\
\hline \multicolumn{4}{|l|}{$\begin{array}{l}\text { Location of primary resusci- } \\
\text { tation }\end{array}$} \\
\hline Other hospitals & $9(47.4)$ & NA & NA \\
\hline Our institute & $10(52.6)$ & NA & NA \\
\hline \multicolumn{4}{|l|}{ Mechanism of injury } \\
\hline Motorbike accident & $7(36.8)$ & NA & NA \\
\hline Car accident & $4(21.1)$ & NA & NA \\
\hline Fall injury (>6 m) & $3(15.8)$ & NA & NA \\
\hline Pedestrian injury & $3(15.8)$ & NA & NA \\
\hline Crushing injury & $2(10.5)$ & NA & NA \\
\hline \multicolumn{4}{|l|}{ Fracture classification $^{\mathrm{a}}$} \\
\hline $61-A$ & $4(21.1)$ & NA & NA \\
\hline $61-\mathrm{B}$ & $6(31.2)$ & NA & NA \\
\hline $61-\mathrm{C}$ & $9(47.4)$ & NA & NA \\
\hline \multicolumn{4}{|l|}{ Faringer zone } \\
\hline I & $11(57.9)$ & NA & NA \\
\hline II & $1(5)$ & NA & NA \\
\hline III & $7(36.8)$ & NA & NA \\
\hline \multicolumn{4}{|l|}{ Jones-Powell classification } \\
\hline 1 & $4(21.1)$ & NA & NA \\
\hline 2 & $4(21.1)$ & NA & NA \\
\hline 3 & $11(57.9)$ & NA & NA \\
\hline \multicolumn{4}{|l|}{ Diversional colostomy } \\
\hline No & $10(52.6)$ & NA & NA \\
\hline Yes & $9(47.4)$ & NA & NA \\
\hline \multicolumn{4}{|l|}{ Arterial embolization } \\
\hline No & $11(57.9)$ & NA & NA \\
\hline Yes & $8(42.1)$ & NA & NA \\
\hline \multicolumn{4}{|l|}{ Treatment protocol } \\
\hline Single-stage treatment & $9(47.4)$ & NA & NA \\
\hline Multi-stage treatments & $10(52.6)$ & NA & NA \\
\hline Numbers of surgery & NA & $5.5(3.6)$ & $5(1-14)$ \\
\hline Admission day & NA & $25.7(13.5)$ & $25(7-67)$ \\
\hline ISS & NA & $23(12.1)$ & $20(9-43)$ \\
\hline NISS & NA & $29(12.0)$ & $27(9-48)$ \\
\hline Mean follow-up (months) & NA & $39.2(8.7)$ & $36(30-63)$ \\
\hline Time to union (months) & NA & $7.0(2.5)$ & $6(4-12)$ \\
\hline
\end{tabular}

${ }^{a}$ The classification of pelvic ring injury is based on Arbeitsgemeinschaft für Osteosynthesefragen classification (2018)

ISS Injury Severity Score, NA not available, NISS New Injury Severity Score, $S D$ standard deviation

nine patients with AO type 61-A, 61-B, and 61-C pelvic ring fractures, respectively. There were 11,1 , and 7 patients with open wounds related to the pelvic fractures located in
Table 3 Associations between concomitant injuries and fracture types of pelvic ring injury

\begin{tabular}{lllll}
\hline Associated injuries & \multicolumn{4}{l}{$\begin{array}{l}\text { Arbeitsgemeinschaft für Osteosynthesefragen } \\
\text { classification for pelvic ring injury }\end{array}$} \\
\cline { 2 - 5 } & $61-\mathrm{A}$ & $61-\mathrm{B}$ & $61-\mathrm{C}$ & $N$ \\
\hline & $\mathrm{A} 1(N=2)$ & $\mathrm{B} 1(N=1)$ & $\mathrm{C} 1(N=5)$ & \\
& $\mathrm{A} 2(N=2)$ & $\mathrm{B} 2(N=4)$ & $\mathrm{C} 2(N=1)$ & \\
& & $\mathrm{B} 3(N=1)$ & $\mathrm{C} 3(N=3)$ & \\
Head & 0 & 0 & 0 & 0 \\
Face & 0 & 1 & 0 & 1 \\
Thorax & 1 & 2 & 3 & 6 \\
Abdomen & 0 & 3 & 1 & 4 \\
Extremity fractures & 1 & 5 & 6 & 12 \\
Spine fracture & 0 & 0 & 2 & 2 \\
Urogenital injury & 0 & 1 & 4 & 5 \\
Rectal injury & 0 & 0 & 5 & 5 \\
Faringer zone & & & & \\
I & 0 & 4 & 7 & 11 \\
II & 1 & 0 & 0 & 1 \\
III & 3 & 2 & 2 & 7 \\
ISS (mean) & 13 & 22 & 28 & NA \\
NISS (mean) & 16 & 30 & 32 & NA \\
\hline
\end{tabular}

CRIF closed reduction and internal fixation; Ex-Fix, external fixator; $I S$, iliosacral screw; $N A$, not available; $O R I F$, open reduction and internal fixation; TITS, trans-iliac-trans-sacral screw

Faringer zones I, II, and III, respectively. All fractures were united at the mean time of $7.0 \pm 2.5$ months.

The associations among concomitant injuries and fracture types are shown in Table 3. The more severe fracture types were accompanied by higher number concomitant injuries, such as extremity fractures, urogenital injury, and rectal injury. Thus, the more severe the fracture, the higher the severity scores.

Table 4 summarizes the treatment methods according to different injury patterns. Conservative treatment (52.6\%) was the most common treatment for anterior pelvic ring injury, followed by percutaneous fixations (36.8\%), including anterior columns screws, pubic screws, external fixator, and anterior superficial internal fixator (ASIF), and then by open reduction and internal fixation (ORIF, 10.5\%). In contrast, for posterior pelvic ring injury, ORIF (47.4\%) was the most common procedure performed using different approaches, followed by percutaneous $(42.1 \%)$ and conservative $(10.5 \%)$ treatments. Percutaneous treatment, either with an external fixator or screws, was the most frequent strategy for the loss of fixation injuries before fracture union.

All the patients completed the radiological and functional outcome evaluations. As shown in Fig. 2, 11 of 19 (57.9\%) and 9 of $19(47.5 \%)$ patients showed excellent reduction quality by Matta/Torenetta and Lefaivre criteria, 
Table 4 Summary of approaches for osteosynthesis and related implants

\begin{tabular}{|c|c|c|c|c|c|c|c|c|c|}
\hline & \multicolumn{9}{|c|}{ Patient number, $N(\%)$} \\
\hline & \multirow[b]{2}{*}{$\begin{array}{l}\text { Conserva- } \\
\text { tive treat- } \\
\text { ment }\end{array}$} & \multirow{2}{*}{$\begin{array}{l}\text { CRIF } \\
\text { Percutaneous } \\
\text { (IS, TITS, } \\
\text { Ex-Fix) }\end{array}$} & \multicolumn{7}{|l|}{ ORIF } \\
\hline & & & $\begin{array}{l}\text { Lateral win- } \\
\text { dow }\end{array}$ & $\begin{array}{l}\text { Dorsal } \\
\text { approach }\end{array}$ & Pfannenstiel & Ilioinguinal & Iliofemoral & $\begin{array}{l}\text { Kocher- } \\
\text { Langen- } \\
\text { beck }\end{array}$ & $\begin{array}{l}\text { Spinopelvic } \\
\text { osteosyn- } \\
\text { thesis }\end{array}$ \\
\hline $\begin{array}{l}\text { Anterior } \\
\text { pelvic ring }\end{array}$ & $10(52.6)$ & $7(36.8)$ & 0 & 0 & $2(10.5)$ & 0 & 0 & NA & NA \\
\hline $\begin{array}{l}\text { Posterior } \\
\text { pelvic ring }\end{array}$ & $2(10.5)$ & $8(42.1)$ & $2(10.5)$ & $2(10.5)$ & NA & $1(5)$ & $1(5)$ & $1(5)$ & $2(10.5)$ \\
\hline $\begin{array}{l}\text { Loss of fixa- } \\
\text { tion }\end{array}$ & NA & $3(15.8)$ & 0 & 0 & 0 & 0 & 0 & 0 & 0 \\
\hline
\end{tabular}

CRIF closed reduction and internal fixation; Ex-Fix external fixator; IS iliosacral screw; NA not available; ORIF open reduction and internal fixation; TITS trans-iliac-trans-sacral screw

Fig. 2 Results of quality of reduction of the pelvic ring by Matta/Torenetta and Lefaivre criteria

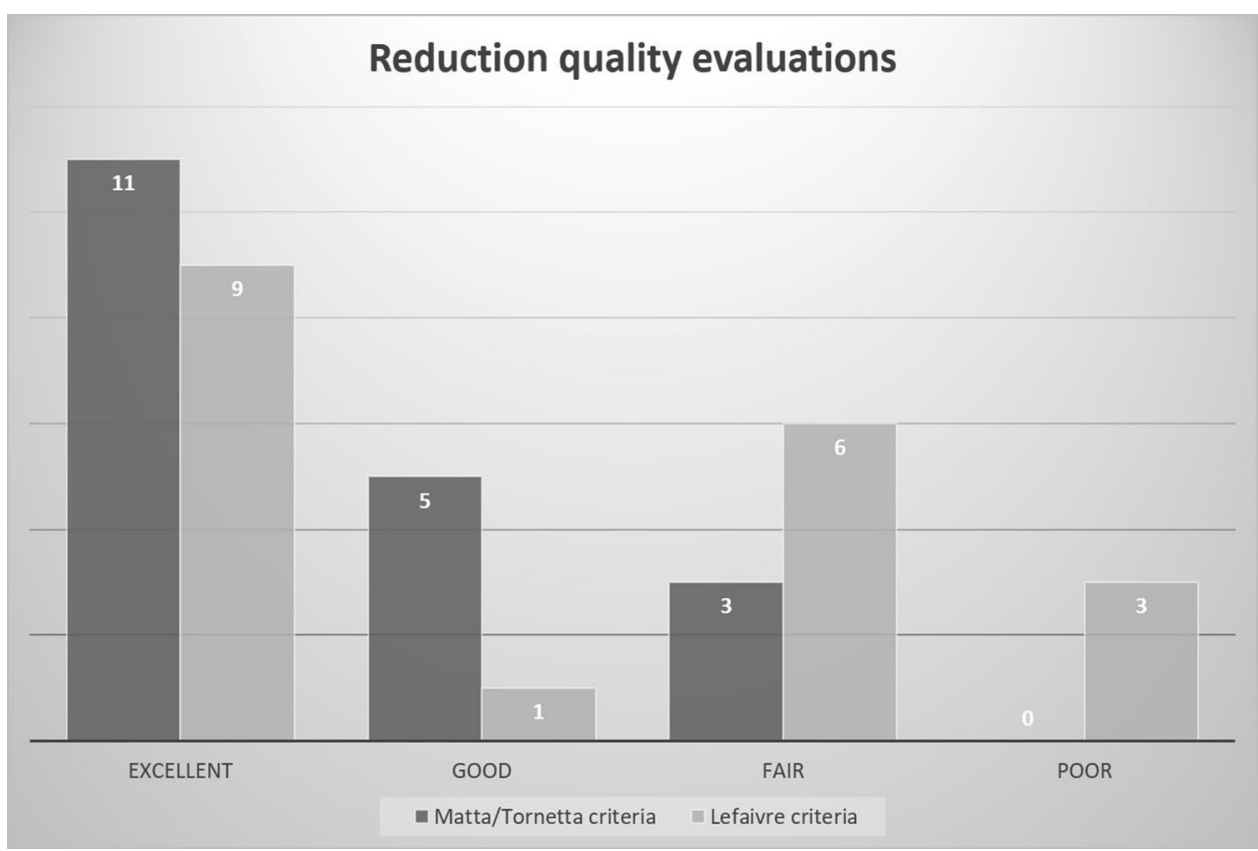

respectively. Functional evaluations were performed at scheduled intervals (Fig. 3). Statistical analysis revealed that the Merle d'Aubigné score improved significantly at each evaluation. However, the improvement stopped from the 24-month to the 36-month evaluation. A similar trend was found for the Majeed hip score. However, there was no significant difference in improvements when comparing the 12- and 24-month, 12- and 36-month, and 24- and 36-month evaluations.

We further analyzed the relationship between the quality of reduction for the pelvic ring and 3-year functional outcomes (Fig. 4). The patients with initial excellent reduction quality of the pelvic ring showed the highest score in both score systems. However, patients with good reduction quality of the pelvic ring $(n=2)$ showed the lowest scores either by Matta/Torenetta or Lefaivre criteria. In addition to open pelvic ring injury, lumbar spine and sacral fractures with lumbosacral plexopathy were also observed, and the symptoms of lumbosacral plexopathy hardly improved, even 3 years after osteosynthesis.

\section{Discussion}

The optimal treatment protocol for pelvic fractures remains debatable. The resuscitation protocol for such injuries should be based on the facilities available at the medical institute, and the aim should be to perform life-saving procedures without causing additional harm to the patient. In this study, 8 of 37 patients with open pelvic fractures died during resuscitation at the ED. Injuries to vital organs, such as the brain, chest cavity, or abdominal cavity, or the open pelvic 


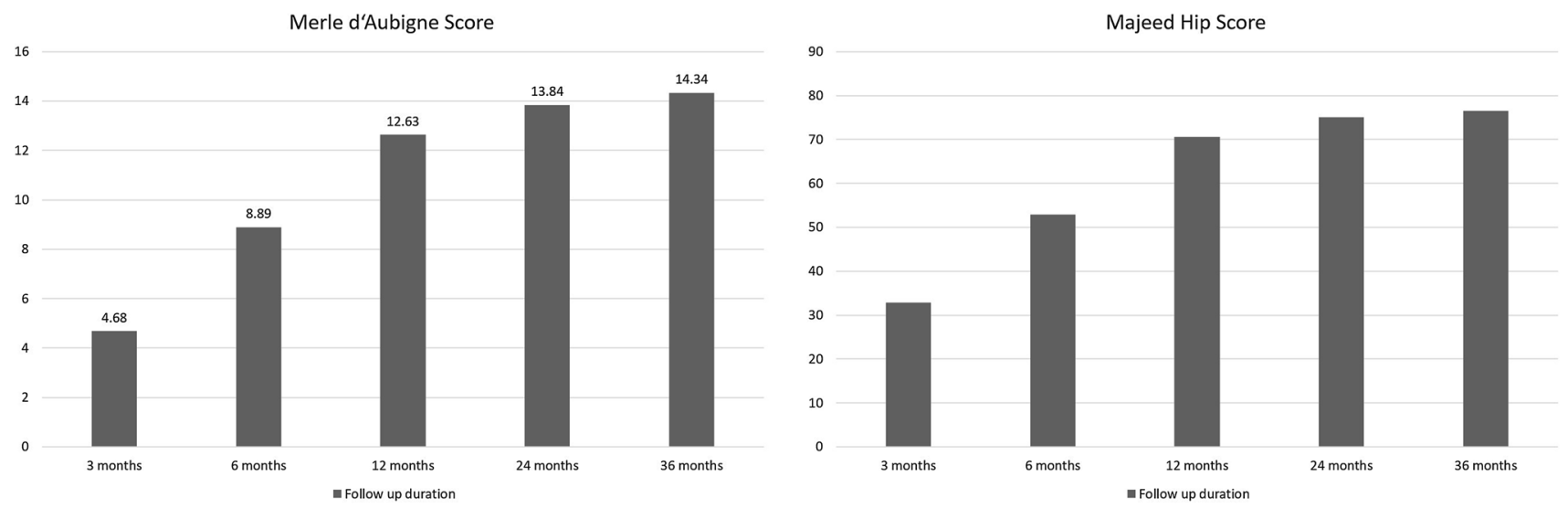

Fig. 3 Results of functional outcome evaluations with Merle d'Aubigné score and Majeed hip score. a Merle d'Aubigné score. b Majeed hip score

(a) 3-year Merle d’Aubigné score

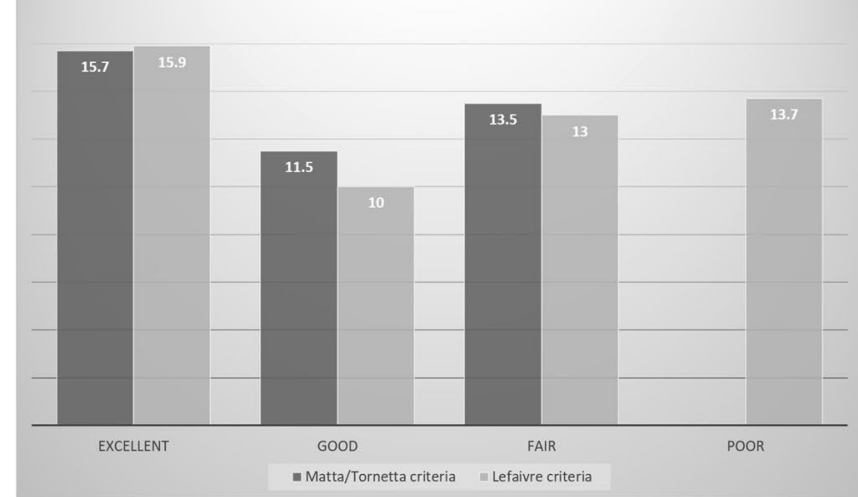

(b)

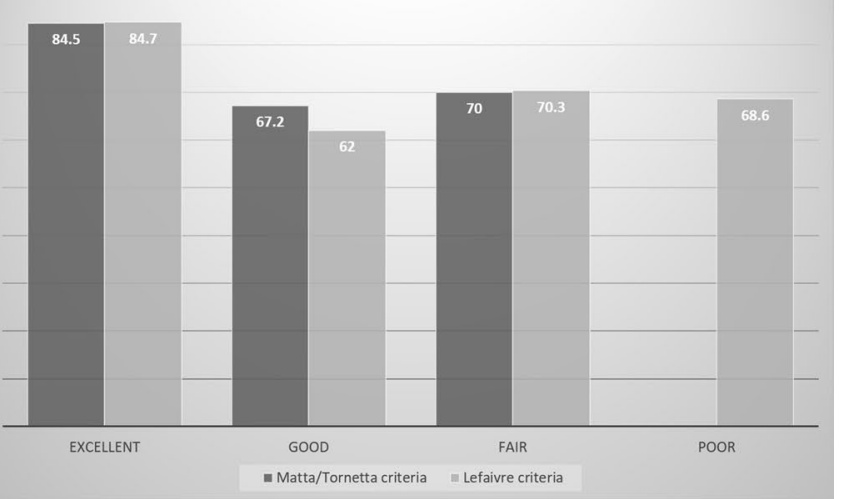

Fig. 4 The relationship between the quality of reduction for the pelvic ring and 3-year functional outcomes. a Merle d'Aubigné score. b Majeed hip score

fracture itself, were the causes of death in these patients. Those who were successfully resuscitated survived the treatment protocol and received complete treatment. Under the protocol for selective diversional colostomies, scheduled debridement surgeries, tailor-made design of osteosynthesis, and individualized rehabilitation protocols, the patients were expected to return to their daily activities, even when the initial functional status was poor.

Open pelvic fracture is a problematical injury not only because of the high mortality rate during the trauma but also due to high infection rates during the treatment [1, 20-22]. The colonized bacteria may originate from contamination by the environment or more likely due to the presence of fecal content in the retroperitoneal cavity. Faringer et al. [8] reported the infection rates in relation to the location of open wound and classified them into zones I, II, and III. They further advocated that all zone I open pelvic fractures should undergo diverting colostomy. Some reports support the statement that diverting colostomy is indicated only for patients with rectal injury despite an open wound that is present in zone I [10, 23, 24]. In our study, there were nine patients (eight with zone I injury and one with zone III injury) who underwent diversional colostomy. The aim of our protocol was to prevent potentially infective sources from reaching the pelvis. The fact is that patients with pelvic fractures might be bed-ridden for a prolonged period, and the wound may get contaminated by fecal matter under this circumstance. For this reason, we believe that fecal contamination, which may lead to infection and sepsis, might be avoided by promptly performing diverting colostomy. Under this protocol, no patient experienced sepsis during hospital stay or chronic osteomyelitis after discharge.

The best surgical strategy to perform osteosynthesis for pelvic fractures remains controversial. The management of the anterior pelvic ring has evolved with the introduction of various types of external fixators, intramedullary implant 
fixation, plate and screw, and ASIF [25-28]. Although various approaches could be used, only two patients received ORIF with plate and screw because of disrupted pubic symphysis through Pfannenstiel incision. In fractures with main wounds located anteriorly, we tried to provide a less aggressive treatment to avoid possible surgical contamination. In contrast, for posterior pelvic ring injury, the treatment policy was more aggressive. Open reduction through various approaches, as mentioned above, aimed to restore the anatomy and stability of the posterior pelvic ring for the purpose of lessening sequelae from prolonged immobilization and malunion. Similar concepts were mentioned by the previous reports for closed pelvic injuries $[4,29$, 30]. We believe that although the possibility of surgical site infection following osteosynthesis for posterior pelvic ring injury exists, adequate stability of the posterior pelvis is still crucial in such patients for early mobilization. As a result of employing a protocol for early mobilization, three cases showed loosening of implants, one with ASIF and two with percutaneous iliosacral screws.

Several possible complications may occur after pelvic fractures, such as gait disturbance, chronic pelvic pain, and functional disability [31, 32]. These complications may be the sum of the complexity of fracture types, surgical approach, and concomitant injuries. Especially in open pelvic fractures, necessary life-saving procedures, such as TAE, limb amputation, and diversional colostomy, may be the cause of temporary functional deficits [25, 33, 34]. Kokubo et al. [7] reported that lower extremity fractures, conservative therapy, and nerve damage affected the 1-year functional outcomes. Additionally, prolonged time for osteosynthesis also led to worse long-term functional outcomes [33]. Among the enrolled patients, fractures in 15 patients were classified as Jones-Powell 2 or 3, with the ISS being 22.5 for AO type B and 28.1 for AO type C. We observed that the functional evaluations during the first 3 months were poor. Although long-term evaluations are lacking, the patients' functions improved initially with each evaluation, but stagnated after 24 months. Additionally, despite good reduction quality evaluated by applied criteria in two patients, they revealed unsatisfactory functional outcomes 36 months after osteosynthesis because of persistent leg numbness, pain, and weakness which are the sequelae of lumbosacral plexopathy after lumbar and sacral fractures. Therefore, we postulated that permanent injuries from severe trauma might have contributed to the stagnation in patients' satisfaction.

Despite our efforts to avoid bias during data collection and analysis, some limitations exist in this study. First, only 19 patients were enrolled in this study. Thus, this cohort is relatively small. However, the incidence of open pelvic fractures is reportedly low, from 2 to $4 \%$ of all pelvic fractures [4], including our study. The considerable rate of mortality among our patients made the enrolled numbers low. Second, although there was a uniform treatment protocol and rehabilitation program for the patients with open pelvic fractures, the locations of the open wound, concomitant injuries, and complexity of fractures were not similar. This diversity among patients' factors might have interfered with the study results. Finally, only two functional evaluations were conducted to evaluate the functional status after trauma and osteosynthesis. Further pivotal clinical studies will benefit from the application of different evaluation tools, such as 36-item short form survey and sexual function assessments, to demonstrate the real functional status of these patients.

\section{Conclusions}

Although the mortality rate in this study was considerable, patients had a good chance of receiving a complete treatment course if they survived the resuscitation. The approaches for the treatment of fractures should be individualized according to the fracture pattern, location of the open wound in the pelvis, and concomitant injuries. Despite the poor initial functional scores, functional improvements may be anticipated. Additionally, an anatomical restoration of the pelvic ring suggested a better functional performance at least a 36-month follow-up. A further study should be conducted to follow these patients in order to obtain long-term functional outcomes.

\section{Acknowledgements None.}

Author Contributions Conceptualization: Y-HY, Y-HH, and I-CT; Investigation: C-HL and I-JC; Writing: Y-HY; Writing-Review and Supervision: Y-CC All authors read and approved the final manuscript.

Funding This research did not receive any specific grant from funding agencies in the public, commercial, or not-for-profit sectors.

Data Availability Statement All data generated or analyzed during this study are included in this published article. The datasets used and/or analyzed during the current study are available from the corresponding author on reasonable request.

\section{Declarations}

Conflict of interest The authors declare that they have no competing interests.

Ethics approval The study was approved by the Institutional Review Board of the hospital (IRB no.: 201900569B0).

Informed Consent Informed consent was obtained from all subjects involved in the study. Written informed consent has been obtained from the patients to publish this paper. 
Open Access This article is licensed under a Creative Commons Attribution 4.0 International License, which permits use, sharing, adaptation, distribution and reproduction in any medium or format, as long as you give appropriate credit to the original author(s) and the source, provide a link to the Creative Commons licence, and indicate if changes were made. The images or other third party material in this article are included in the article's Creative Commons licence, unless indicated otherwise in a credit line to the material. If material is not included in the article's Creative Commons licence and your intended use is not permitted by statutory regulation or exceeds the permitted use, you will need to obtain permission directly from the copyright holder. To view a copy of this licence, visit http://creativecommons.org/licenses/by/4.0/.

\section{References}

1. Tseng IC, Chen IJ, Chou YC, Hsu YH, Yu YH (2020) Predictors of acute mortality after open pelvic fracture: experience from 37 patients from a Level I Trauma Center. World J Surg 44:3737-3742. https://doi.org/10.1007/s00268-020-05675-z

2. Brenneman FD, Katyal D, Boulanger BR, Tile M, Redelmeier DA (1997) Long-term outcomes in open pelvic fractures. J Trauma 42:773-777. https://doi.org/10.1097/00005373-19970 5000-00003

3. Ferrera PC, Hill DA (1999) Good outcomes of open pelvic fractures. Injury 30:187-190. https://doi.org/10.1016/s00201383(98)00246-0

4. Grotz MR, Allami MK, Harwood P, Pape HC, Krettek C, Giannoudis PV (2005) Open pelvic fractures: epidemiology, current concepts of management and outcome. Injury 36:1-13. https:// doi.org/10.1016/j.injury.2004.05.029

5. Katsoulis E, Giannoudis PV (2006) Impact of timing of pelvic fixation on functional outcome. Injury 37:1133-1142. https:// doi.org/10.1016/j.injury.2006.07.017

6. Kabak S, Halici M, Tuncel M, Avsarogullari L, Baktir A, Basturk M (2003) Functional outcome of open reduction and internal fixation for completely unstable pelvic ring fractures (type C): a report of 40 cases. J Orthop Trauma 17:555-562. https:// doi.org/10.1097/00005131-200309000-00003

7. Kokubo Y, Oki H, Sugita D, Takeno K, Miyazaki T, Negoro K, Nakajima H (2017) Functional outcome of patients with unstable pelvic ring fracture. J Orthop Surg (Hong Kong) 25:2309499016684322. https://doi.org/10.1177/2309499016 684322

8. Faringer PD, Mullins RJ, Feliciano PD, Duwelius PJ, Trunkey DD (1994) Selective fecal diversion in complex open pelvic fractures from blunt trauma. Arch Surg 129:958-963; discussion 963. https://doi.org/10.1001/archsurg.1994.0142033007 2014

9. Baker SP, O'Neill B, Haddon W, Long WB (1974) The Injury Severity Score: a method for describing patients with multiple injuries and evaluating emergency care. J Trauma 14(3):187196. https://doi.org/10.1097/00005373-197403000-00001

10. Copes WS, Champion HR, Sacco WJ, Lawnick MM, Keast SL, Bain LW (1988) The injury severity score revisited. J Trauma 28(1):69-77. https://doi.org/10.1097/00005373-19880 1000-00010

11. AO/OTA fracture and dislocation classification compendium (2018). https://classification.aoeducation.org/

12. Jones AL, Powell JN, Kellam JF, McCormack RG, Dust W, Wimmer P (1997) Open pelvic fractures. a multicenter retrospective analysis. Orthop Clin North Am 28:345-350. https:// doi.org/10.1016/s0030-5898(05)70293-5

13. Mataliotakis GI, Giannoudis PV (2011) Radiological measurements for postoperative evaluation of quality of reduction of unstable pelvic ring fractures: advantages and limitations. Injury 42:1395-1401. https://doi.org/10.1016/j.injury.2011.10.012

14. Lefaivre KA, Blachut PA, Starr AJ, Slobogean GP, O'Brien PJ (2014) Radiographic displacement in pelvic ring disruption: reliability of 3 previously described measurement techniques. J Orthop Trauma 28:160-166. https://doi.org/10.1097/BOT. 0b013e31829efcc5

15. Yu YH, Liu CH, Hsu YH, Chou YC, Chen IJ, Wu CC (2021) Matta's criteria may be useful for evaluating and predicting the reduction quality of simultaneous acetabular and ipsilateral pelvic ring fractures. BMC Musculoskelet Disord 22:544. https:// doi.org/10.1186/s12891-021-04441-z

16. D'Aubigne RM, Postel M (1954) Functional results of hip arthroplasty with acrylic prosthesis. J Bone Joint Surg Am 36-A:451-475. https://doi.org/10.2106/00004623-19543 6030-00001

17. d'Aubigné RM, Postel M (2009) The classic: functional results of hip arthroplasty with acrylic prosthesis. 1954. Clin Orthop Relat Res 467:7-27. https://doi.org/10.1007/s11999-008-0572-1

18. Lefaivre KA, Slobogean GP, Valeriote J, O'Brien PJ, Macadam SA (2012) Reporting and interpretation of the functional outcomes after the surgical treatment of disruptions of the pelvic ring: a systematic review. J Bone Joint Surg Br 94:549-555. https://doi.org/10.1302/0301-620X.94B4.27960

19. Majeed SA (1989) Grading the outcome of pelvic fractures. J Bone Joint Surg Br 71:304-306. https://doi.org/10.1302/0301620X.71B2.2925751

20. Hermans E, Edwards MJR, Goslings JC, Biert J (2018) Open pelvic fracture: the killing fracture? J Orthop Surg Res 13:83. https://doi.org/10.1186/s13018-018-0793-2

21. Fitzgerald CA, Moore TJ Jr, Morse BC, Subramanian A, Dente CJ, Patel DC, Reisman WM, Schenker ML, Gelbard RB (2017) The role of diverting colostomy in traumatic blunt open pelvic fractures. Am Surg 83:e280-e282. https://doi.org/10.1177/ 000313481708300805

22. Giordano V, Koch HA, Gasparini S, Serrão de Souza F, Labronici PJ, do Amaral NP (2016) Open pelvic fractures: review of 30 cases. Open Orthop J 10:772-778. https://doi.org/10.2174/ 1874325001610010772

23. Govaert G, Siriwardhane M, Hatzifotis M, Malisano L, Schuetz M (2012) Prevention of pelvic sepsis in major open pelviperineal injury. Injury 43:533-536. https://doi.org/10.1016/j.injury. 2011.12.002

24. Van Wessem KJP, Mackay PJ, King KL, Balogh ZJ (2012) Selective faecal diversion in open pelvic fractures: reassessment based on recent experience. Injury 43:522-525. https:// doi.org/10.1016/j.injury.2011.09.001

25. Wright RD Jr (2018) Indications for open reduction internal fixation of anterior pelvic ring disruptions. J Orthop Trauma 32(Supplement 6):S18-S23. https://doi.org/10.1097/BOT.00000 00000001252

26. Lai CY, Liu CH, Hsu YH, Yeh WL, Chou YC, Wu CC, Yu YH (2018) Retrograde intramedullary titanium elastic nail fixation: an alternative to traditional osteosynthesis surgery for patients with superior pubic ramus fracture. Formos J Musculoskelet Disord 9:145-152

27. Wojahn RD, Gardner MJ (2019) Fixation of anterior pelvic ring injuries. J Am Acad Orthop Surg 27:667-676. https://doi.org/ 10.5435/JAAOS-D-17-00839

28. Vaidya R, Woodbury D, Nasr K (2018) Anterior subcutaneous internal pelvic fixation/INFIX: a systemic review. J Orthop Trauma 32(Supplement 6):S24-S30. https://doi.org/10.1097/ BOT.0000000000001248

29. Vaidya R, Colen R, Vigdorchik J, Tonnos F, Sethi A (2012) Treatment of unstable pelvic ring injuries with an internal anterior fixator and posterior fixation: initial clinical series. 
J Orthop Trauma 26:1-8. https://doi.org/10.1097/BOT.0b013 e318233b8a7

30. Verbeek DO, Routt ML Jr (2018) High-energy pelvic ring disruptions with complete posterior instability: contemporary reduction and fixation strategies. J Bone Joint Surg Am 100:1704-1712. https://doi.org/10.2106/JBJS.17.01289

31. Meyhoff CS, Thomsen CH, Rasmussen LS, Nielsen PR (2006) High incidence of chronic pain following surgery for pelvic fracture. Clin J Pain 22:167-172. https://doi.org/10.1097/01.ajp.00001 74266.12831.a2

32. Miranda MA, Riemer BL, Butterfield SL, Burke CJ 3rd (1996) Pelvic ring injuries. a long term functional outcome study. Clin Orthop Relat Res 329:152-159. https://doi.org/10.1097/00003 086-199608000-00019

33. Penn-Barwell JG (2011) Outcomes in lower limb amputation following trauma: a systematic review and meta-analysis. Injury 42:1474-1479. https://doi.org/10.1016/j.injury.2011.07.005
34. Lai CY, Tseng IC, Su CY, Hsu YH, Chou YC, Chen HW, Yu YH (2020) High incidence of surgical site infection may be related to suboptimal case selection for non-selective arterial embolization during resuscitation of patients with pelvic fractures: a retrospective study. BMC Musculoskelet Disord 21:335. https://doi.org/10. 1186/s12891-020-03372-5

Publisher's Note Springer Nature remains neutral with regard to jurisdictional claims in published maps and institutional affiliations. 